\title{
Evolution du capteur électronique pour la dosimétrie des neutrons développé au LEPOFI
}

\author{
M. BARELAUD*, B. DUBARRY-CHABANAIS*, D. PAUL*, \\ L. MAKOVICKA*, J.L. DECOSSAS*, J.C. VAREILLE*
}

RÉSUMÉ La dosimétrie neutronique des personnels à l'aide de dispositifs électroniques est étudlée depuis quelques années. Ces dispositifs offrent un avantage majeur : l'obtention d'une réponse en temps réel. Après une étude de faisabilité d'un système à double diode, fondé sur l'utilisation de la méthode différentielle, le capteur (convertisseur de polyéthylène $\left(\mathrm{CH}_{2}\right)_{n}$ avec une implantation d'ions ${ }^{10} \mathrm{~B}$ et diode silicium) a été expérimenté dans divers champs de neutrons et modélisé afin de confirmer et compléter les mesures. La contribution des photons $\gamma$ de ces champs de neutrons est la principale perturbation pour la détermination de la réponse aux neutrons. La sensibilité aux neutrons $S_{n}$ est de 1 Impulsion $\mathrm{cm}^{-2} \mu \mathrm{Sv}^{-1}$ pour les thermiques et sur la gamme d'énergie 1,5 - $5 \mathrm{MeV}$ avec un seuil énergétique à $125 \mathrm{keV}$ pour les particules secondaires détectées. Lorsque les équivalents de dose $\gamma$ et neutron (équivalent de dose ambient $H^{\star}(10)$ ) deviennent du même ordre de grandeur, la perte de sensibilité $S_{n}$ est d'autant plus grande que la composante du spectre neutronique en dessous de $500 \mathrm{keV}$ est plus importante par rapport à celle au-dessus de cette énergie. La sensibilité aux photons $\gamma\left(S_{\gamma}\right)$ est de l'ordre de 300 impulsions $\mathrm{cm}^{-2} \mu \mathrm{Sv}^{-1}$ avec un seuil de coupure à $130 \mathrm{keV}$. La modélisation du capteur a été effectuée avec un code Monte-Carlo (EGS4) pour les photons $\gamma$ et un programme développé au laboratoire (PNEDIOD) pour les neutrons. Une structure adéquate utilisant tous les paramètres optimisés issus de la modélisation améliorerait la discrimination $(n-\gamma)$ en divisant la sensibilité $S_{\gamma}$ par 15 . Le seuil de détection énergétique de notre système pour les neutrons serait alors inférieur à $200 \mathrm{keV}$.

ABSTRACT Personnel neutron dosimetry using electronic devices has been studied for many years. These devices present a major advantage,viz a real time response. After a feasibility study of a double diode device using the differential method, the sensor (boron 10 implanted polyethylene $\left(\mathrm{CH}_{2}\right)_{n}$ converter and silicon diode) was experimented in many neutron fields; a mathematical model was used in order to confirm and complete measurements. The contribution of photons $\gamma$ in these neutron fields is the main perturbation to determine the neutron response. The neutron sensitivity $S_{n}$ is about 1 pulse $\mathrm{cm}^{-2} \mu \mathrm{Sv}^{-1}$ for thermal neutrons and in the energy range $1.5-5 \mathrm{MeV}$ with an energy threshold at $125 \mathrm{keV}$ for secondary particles. When neutron and photon dose equivalents (ambient dose equivalent $H^{*}(10)$ ) reach the same magnitude, the loss of neutron sensitivity $S_{n}$ is the greater as the component of the neutron spectrum under $500 \mathrm{keV}$ is greater. Photon sensitivity $S_{\gamma}$ is about 300 pulses $\mathrm{cm}^{-2} \mu \mathrm{Sv}^{-1}$ with an energy threshold at $130 \mathrm{keV}$. The modelisation of sensor was realized with a Monte-Carlo program (EGS4) for photons $\gamma$ and a LEPOFI program (PNEDIOD) for neutrons. Thanks to

* Laboratoire d'électronique des polymères sous faisceaux ioniques (LEFOPI), Faculté des sciences de Limoges, 123 avenue Albert Thomas, 87060 Limoges Cedex, France.

* Université de Franche-Comté, IUT Belfort-Montbéliard, rue Engel Gros, BP 527, 90016 Belfort Cedex, France. 
an appropriate structure using optimised parameters, the (n- $\gamma$ ) discrimination will be improved, because of a photon sensitivity $S_{\gamma}$ divided by 15 . Under these conditions, the energy threshold of our device to neutrons will thus be lower than $200 \mathrm{keV}$.

\section{Introduction}

Une étude préliminaire de faisabilité permettant d'effectuer une dosimétrie neutronique individuelle à l'aide d'un dispositif actif a été réalisée au laboratoire [1] dans la période 1987-89. Le schéma de principe du dispositif est présenté sur la figure 1. La réponse est composée des grandeurs d'entrée désirées $\left(G_{d}\right)$ d'une part et de celles interférentes $\left(G_{i}\right)$ d'autre part. Toutes deux, soumises à des fluctuations statistiques, peuvent varier en fonction des grandeurs modifiantes $\left(G_{m}\right)$. Afin de s'affranchir en partie de la contribution des grandeurs interférentes $\left(G_{i}\right)$ nous avons appliqué la méthode différentielle qui a été proposée par le laboratoire et utilisée par Makovicka [8]. A partir des résultats obtenus lors de la phase préliminaire, des études à caractère fondamental ont été développées de manière à décrire théoriquement les interactions des neutrons [5] et des photons $\gamma$ [11] sur les dosimètres électroniques. En parallèle, nous avons effectué des mesures expérimentales qui confirment les résultats de la simulation sur notre modèle. Nous présentons ici d'une part les résultats relatifs à la perturbation apportée par des photons $\gamma$ dans notre dosimètre neutronique à diodes et d'autre part les différentes réponses délivrées par le capteur lorsqu'il est soumis à divers champs de neutrons.

A partir des résultats acquis lors de l'étude de faisabilité [2] et des études fondamentales, nous donnons des comparaisons permettant de mettre à jour les évolutions du capteur ; par la suite, les conclusions que nous apportons ont permis de définir et de proposer un dispositif de "deuxième génération" plus proche des exigences de la dosimétrie individuelle, dans le cadre des recommandations internationales.

Ce nouveau capteur - actuellement à l'étude - nous amène à explorer d'autres voies de conception étroitement liées à la microélectronique.

\section{Perturbation de la réponse du capteur dues aux photons $\gamma$}

Pour des raisons évidentes dues à la nature du matériau utilisé dans les détecteurs (silicium), notre dispositif s'avère être sensible à la présence des photons $\gamma$. 


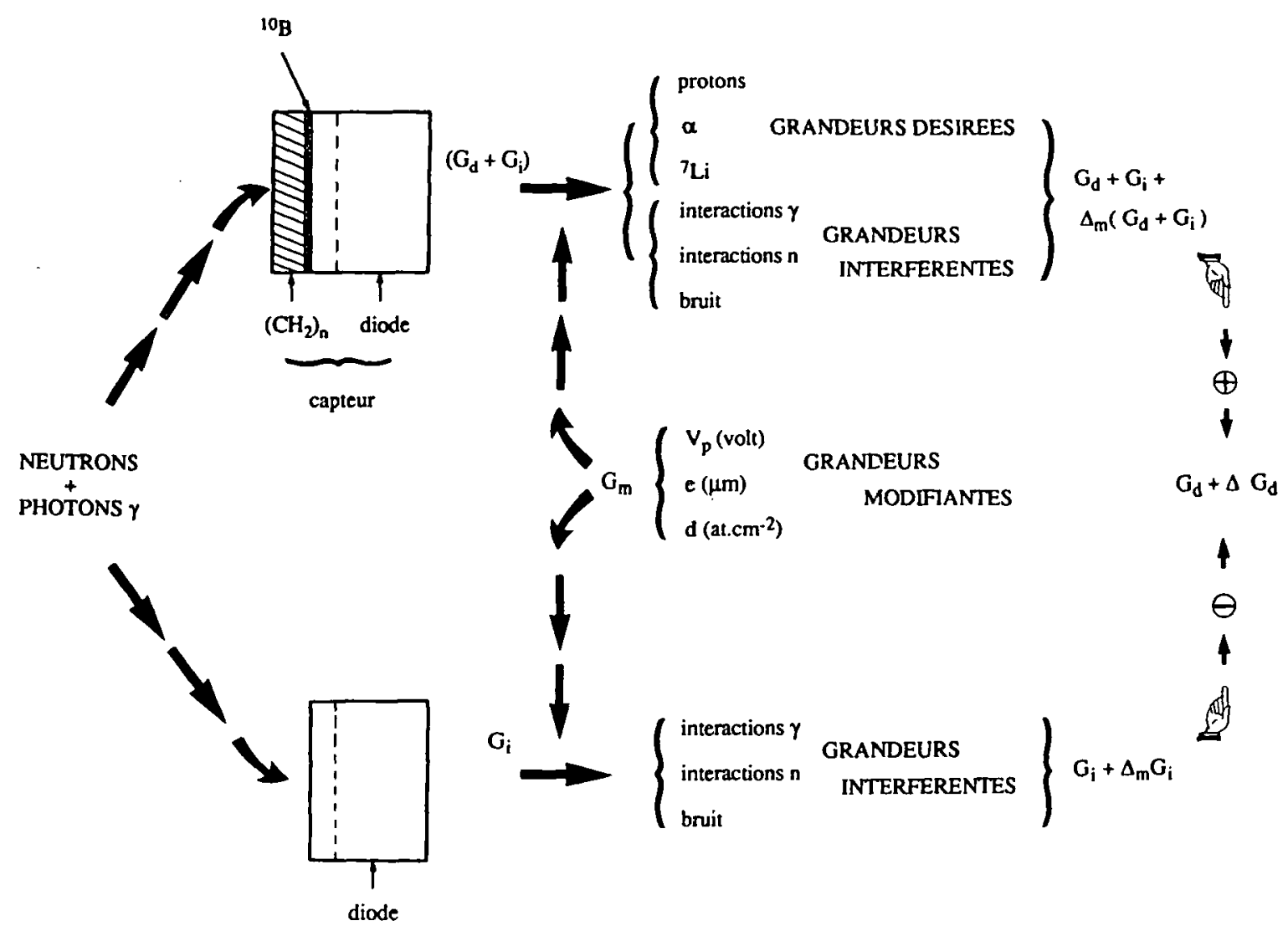

Fig. 1. - Principe du systeme utilisé. Schematic diagram of the system.

Afin d'améliorer, voire optimiser, la réponse attendue aux neutrons, il est nécessaire de caractériser la réponse aux photons. Cette séparation des composantes a été mise en équation [11] :

$$
R_{n, \gamma}=R_{n}+R_{\gamma}=S_{\gamma} \cdot H_{\gamma}+S_{n} \cdot H_{n}=S_{\gamma}\left(H_{\gamma} / H_{n}\right) \cdot H_{n}+S_{n} \cdot H_{n}
$$

avec $R_{n, \gamma}$ la réponse totale du dosimètre, $R_{\gamma}, R_{n}, S_{\gamma}$ et $S_{n}$ les réponses et sensibilités respectives (impulsions $\mathrm{cm}^{-2} \mu \mathrm{Sv}^{-1}$ ) aux photons $\gamma$ et aux neutrons, $\mathrm{H}_{\gamma}$ et $\mathrm{H}_{\mathrm{n}}$ les équivalents de dose respectifs en $\gamma$ et en neutrons.

Cette sensibilité $S_{\gamma}$ peut être favorable ou défavorable suivant le type de dosimètre que nous souhaitons réaliser. Nécessaire dans le cas d'un dosimètre mixte $(n-\gamma)$, c'est une perturbation dans le cas d'un détecteur de neutrons seuls. Dans les deux cas, une connaissance approfondie de la sensibilité du système aux photons $\gamma$ est indispensable pour l'exploitation du dosimètre.

\subsection{Mesures expérimentales et intercomparaison}

Des sources de ${ }^{60} \mathrm{Co}$ et de ${ }^{137} \mathrm{Cs}$ ont permis de réaliser nos irradiations en photons $\gamma$. Durant l'étude de faisabilité, nous avons utilisé comme détecteur une diode ENERTEC avec une zone désertée de 
$80 \mu \mathrm{m}$ de profondeur. Compte tenu de nos objectifs et des résultats obtenus, une première évolution a amené à l'utilisation de détecteurs avec des profondeurs de zone désertée plus faibles (au voisinage de $30 \mu \mathrm{m}$ ).

\section{a) Influence du débit d'équivalent de dose}

Pour ces détecteurs ENERTEC qui sont des diodes au silicium implantées et passivées, l'étude relative à l'influence du débit d'équivalent de dose sur la réponse a abouti aux conclusions suivantes :

- pour des débits d'équivalents de dose $\gamma$ compris entre $10 \mu \mathrm{Sv} / \mathrm{h}$ et $10 \mathrm{mSv} / \mathrm{h}$, la réponse obtenue est indépendante du débit ;

- au-dessus de $10 \mathrm{mSv} / \mathrm{h}$, les limites électroniques du système font apparaître des phénomènes d'empilement qui déforment le spectre énergétique et entraînent une surestimation de la réponse ;

- en dessous de $10 \mu \mathrm{Sv} / \mathrm{h}$, nous nous trouvons confrontés aux problèmes des faibles équivalents de dose et au fait que le mouvement propre du détecteur devient prépondérant par rapport au signal, d'où l'importance d'une bonne connaissance du rapport signal/bruit qui correspond, dans notre cas à :

$$
\frac{S}{N}=\frac{N_{T}-M_{P}}{M_{P}} \text { avec } N=M_{P}
$$

$\mathrm{N}_{\mathrm{T}}=$ nombre total d'impulsions enregistrées, le détecteur étant soumis à une source de photons $\gamma$,

$M_{p}$ (mouvement propre) $=$ nombre total d'impulsions enregistrées en l'absence de toute source.

\section{b) Sensibilité $S_{\gamma}$ du dosimètre}

Les résultats présentés ont été obtenus avec des diodes au silicium implantées et passivées, fabriquées par CANBERRA suivant la technologie "planar" (PIPS : passivated implanted planar silicon). Elles ont une surface active de $150 \mathrm{~mm}^{2}$ et sont polarisées sous $15 \mathrm{~V}$, ce qui correspond à une zone désertée de $30 \mu \mathrm{m}$, dans l'obscurité et à température ambiante. Les réponses sont données en fonction du seuil de coupure utilisé pour l'intégration des spectres. Cette coupure est nécessaire au niveau de la définition des termes "sensibilité $S_{\gamma}$ " (impulsions $\mathrm{cm}^{-2}$ $\mu S v^{-1}$ ) ou "réponse" (impulsions $\mu S v^{-1}$ ) qui ne prennent en compte que les impulsions générées par les photons $\gamma$. Nous avons montré que les 15 premiers canaux (130 keV) correspondent à la majeure partie du bruit électronique. En fixant le seuil de coupure à $130 \mathrm{keV}$, la réponse au ${ }^{60} \mathrm{Co}$ comme au ${ }^{137} \mathrm{Cs}$ est de l'ordre de 480 impulsions $\mu \mathrm{Sv}^{-1}$.

\section{c) Comparaison avec d'autres dispositifs électroniques}

Eisen a proposé en 1984 [6] un système utilisant une diode à barrière de surface ORTEC ; Matsumoto [9] en 1991 a utilisé une diode PIN comme capteur sur son dosimètre. Nous comparons les performances de 
notre dispositif à celles des deux systèmes cités ci-dessus. Le tableau I fait apparaître les différentes sensibilités en fonction : du type de détecteur, de la profondeur de zone désertée, de la surface active, du seuil de coupure énergétique.

TABLEAU I

Sensibilité aux photons $\gamma\left(S_{\gamma}\right)$ de différents dosimètres à semiconducteur Photon sensitivities $\mathbf{S}_{\gamma}$ of various semiconductor dosemeters

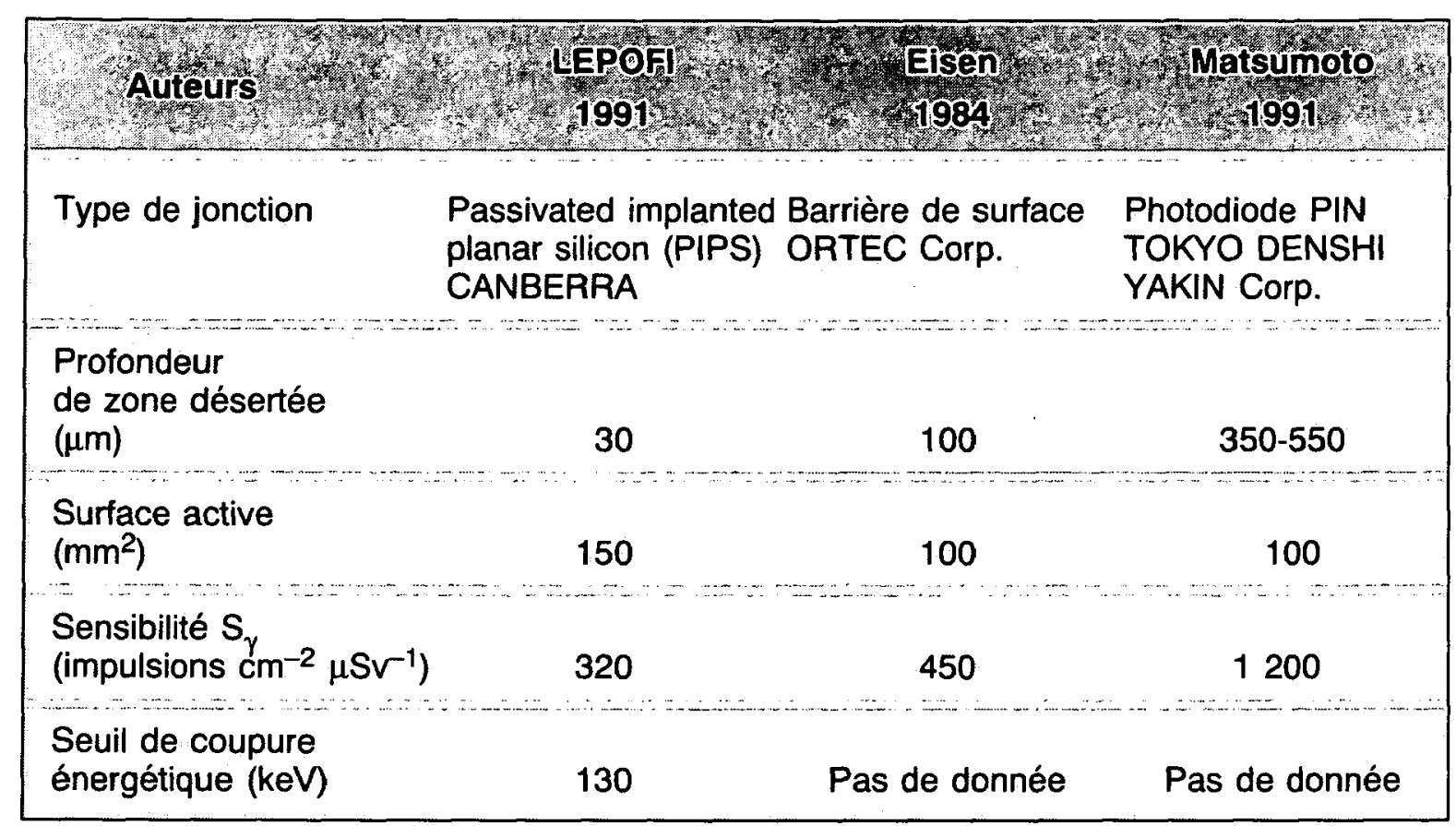

Nous constatons, d'après ce tableau, que notre système répond bien à l'objectif que nous nous étions fixé pour la détection des neutrons, à savoir une sensibilité minimale aux photons $\gamma$, en l'occurrence la plus faible parmi les références citées, soit :

$$
\mathrm{S}_{\gamma}=320 \text { impulsions } \mathrm{cm}^{-2} \mu \mathrm{Sv}^{-1}
$$

\subsection{Modélisation du capteur et simulation}

L'optimisation du système passe par la compréhension des phénomènes physiques entrant dans la réponse globale du dispositif et l'étude théorique de cette réponse dans diverses configurations. Certaines expérimentations étant difficiles voire impossibles à mettre en œuvre, le calcul offre une grande souplesse dans l'étude des paramètres en jeu.

\section{a) Simulation du transport photons-électrons par les méthodes de Monte-Carlo}

Dans le domaine énergétique et les milieux qui nous concernent, le nombre d'interactions subies par un photon est faible alors qu'il est élevé pour les électrons. Par exemple, si un photon de quelques MeV subit une trentaine d'interactions, l'électron d'énergie comparable en connaît 
10000 fois plus [11]. L'importance du nombre d'événements a conduit à développer des théories de diffusion multiple qui déterminent le résultat global d'un certain nombre d'interactions sous forme de distributions des déflexions angulaires et des pertes d'énergies. Ces théories sont à la base des codes macroscopiques utilisant une variante de la méthode de Monte-Carlo pour le transport des électrons.

D. Paul a donc introduit au laboratoire le code EGS4 [10] (electron gamma shower 4). De nombreuses publications relatives aux applications dosimétriques de ce code macroscopique de type Monte-Carlo nous ont permis de modéliser la structure de notre capteur. Les processus physiques pris en compte par ce code sont :

- pour les photons : la diffusion cohérente, la diffusion Compton, l'effet photoélectrique et la production de paires;

- pour les électrons : la diffusion de Möller $\left(e^{-} e^{-}\right)$et de Bhabha $\left(e^{+} e^{-}\right)$, l'interaction coulombienne sur les noyaux suivant la théorie de diffusions multiples de Molière et, enfin, la production de rayonnement de freinage.

La gamme d'énergie possible s'étend, pour les photons, du keV à plusieurs milliers de $\mathrm{GeV}$ et, pour les électrons, de la dizaine de keV à plusieurs milliers de GeV.

\section{b) Les résultats de la simulation}

Les premiers résultats obtenus ont permis de comparer les spectres d'impulsions expérimentaux et théoriques en ${ }^{60} \mathrm{Co}$ (fig. 2) et les sensibilités pour une irradiation en ${ }^{60} \mathrm{Co}$ pour divers seuils de coupure énergétique (Tab. II).

TABLEAU II

Comparaison des sensibilités $\mathbf{S}_{\gamma}$ expérimentale et théorique en fonction du seuil de coupure

Experimental and computed sensitivities $S_{\gamma}$ vs energetic thresholds

\begin{tabular}{|c|c|c|}
\hline $\begin{array}{l}\text { Seuil de } \\
\text { energétiqu } \\
\text { enter }\end{array}$ & ensib & usv \\
\hline 0 & 980 & 810 \\
\hline 130 & 134 & 320 \\
\hline
\end{tabular}

Toujours par simulation, nous avons pu confirmer que l'augmentation de la zone désertée engendrait une augmentation de la sensibilité $S_{\gamma}$ On obtient un facteur multiplicatif de 1,2 (valeur expérimentale 1,4) pour $S_{\gamma}$ lorsque l'on passe de $30 \mu \mathrm{m}$ à $90 \mu \mathrm{m}$. 
Par la suite, nous avons montré que la sensibilité $S_{\gamma}$ ne variait pas très fortement (fig. 3) en fonction de l'énergie des photons $\gamma$. On ne peut pas parler d'indépendance énergétique, mais si l'on prend en compte la sensibilité moyenne des 5 points de la figure 3, soit 940 impulsions $\mathrm{cm}^{-2} \mu \mathrm{Sv}^{-1}$ sur toute la gamme d'énergie, nous travaillons alors avec une incertitude de $\pm 30 \%$.

Enfin et comme évoqué dans l'introduction, le dosimètre placé dans un champ mixte $(n-\gamma)$ répond à la fois aux photons $\gamma$ et aux neutrons. Afin de s'affranchir en partie de cette contribution $\gamma$, nous utilisons la méthode différentielle [8]. Cette dernière trouve ses limites de validité lorsque la sensibilité $S_{\gamma}$ devient très grande devant $S_{n}$, c'est-à-dire vers les faibles énergies. Dans l'état actuel, si nous voulons des sensibilités $S_{\gamma}$ et $S_{n}$ du même ordre de grandeur (environ 1 impulsion $\mathrm{cm}^{-2} \mu \mathrm{Sv}^{-1}$ ) les résultats du calcul montre que le seuil de coupure énergétique doit être fixé à $300 \mathrm{keV}$. Malheureusement, ce seuil peut amener des distorsions de réponse aux neutrons. Aussi une amélioration sensible seraitelle de réduire la sensibilité du dispositif aux photons $\gamma$. C'est ce que nous avons montré par simulation. La figure 4 compare le spectre des impulsions délivrées, sous faisceau de photons $\gamma$, d'une part par le dosimètre actuel et d'autre part par un dosimètre dans lequel toutes les parties métalliques sont supprimées. La sensibilité $S_{\gamma}$ est alors divisée par un facteur 13. La réalisation d'un tel dispositif est en cours.

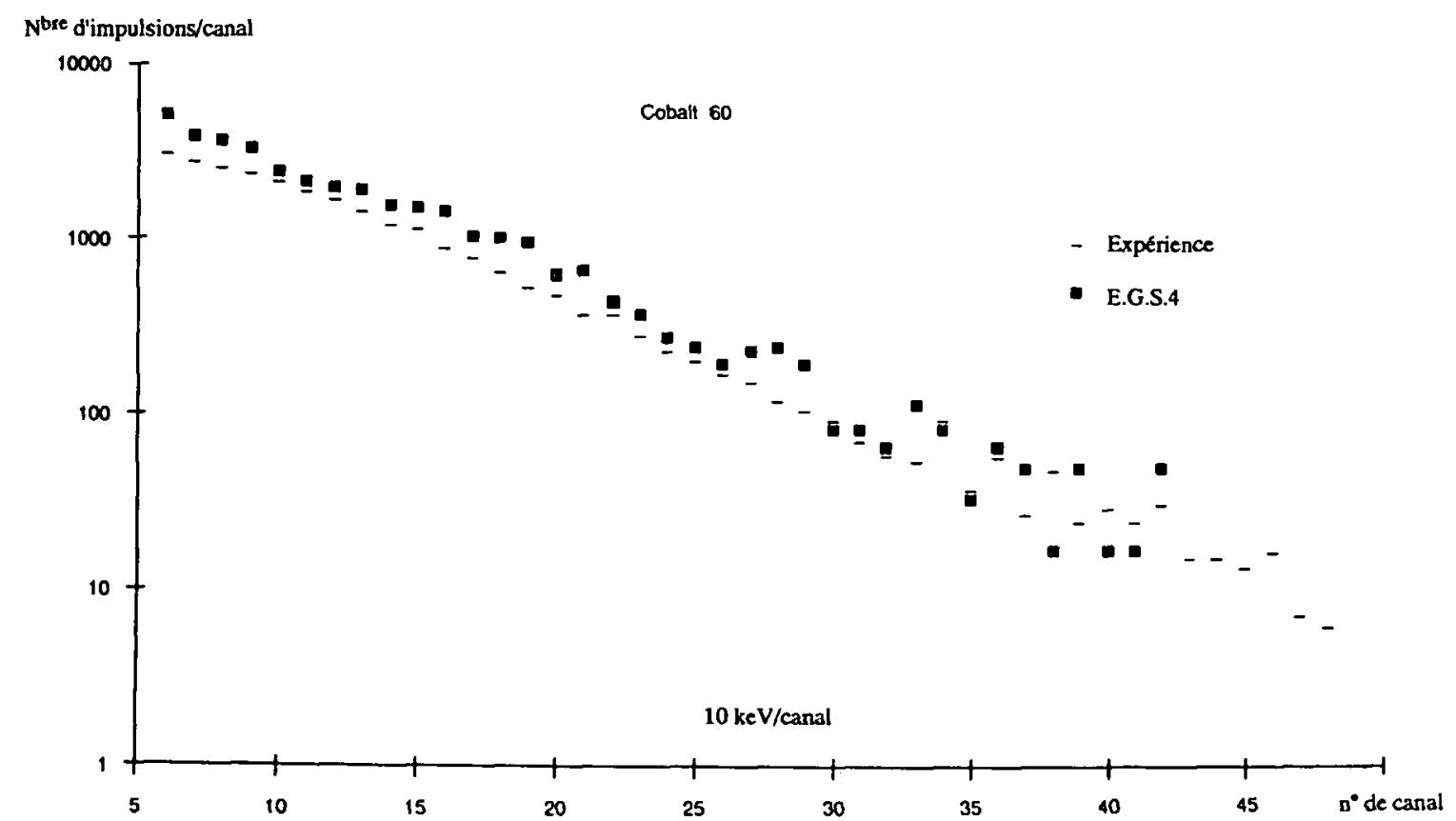

Fig. 2. - Spectre d'impulsions issu du capteur (expérimental et théorique); $H=100 \mu S v$; profondeur de zone désertée $=30 \mu \mathrm{m} ; E_{\gamma}=1,25 \mathrm{MeV}$.

${ }^{60} \mathrm{Co}$ experimental and computed spectra with a depleted layer of $30 \mu \mathrm{m} ; H=$ $100 \mu \mathrm{Sv}$. 


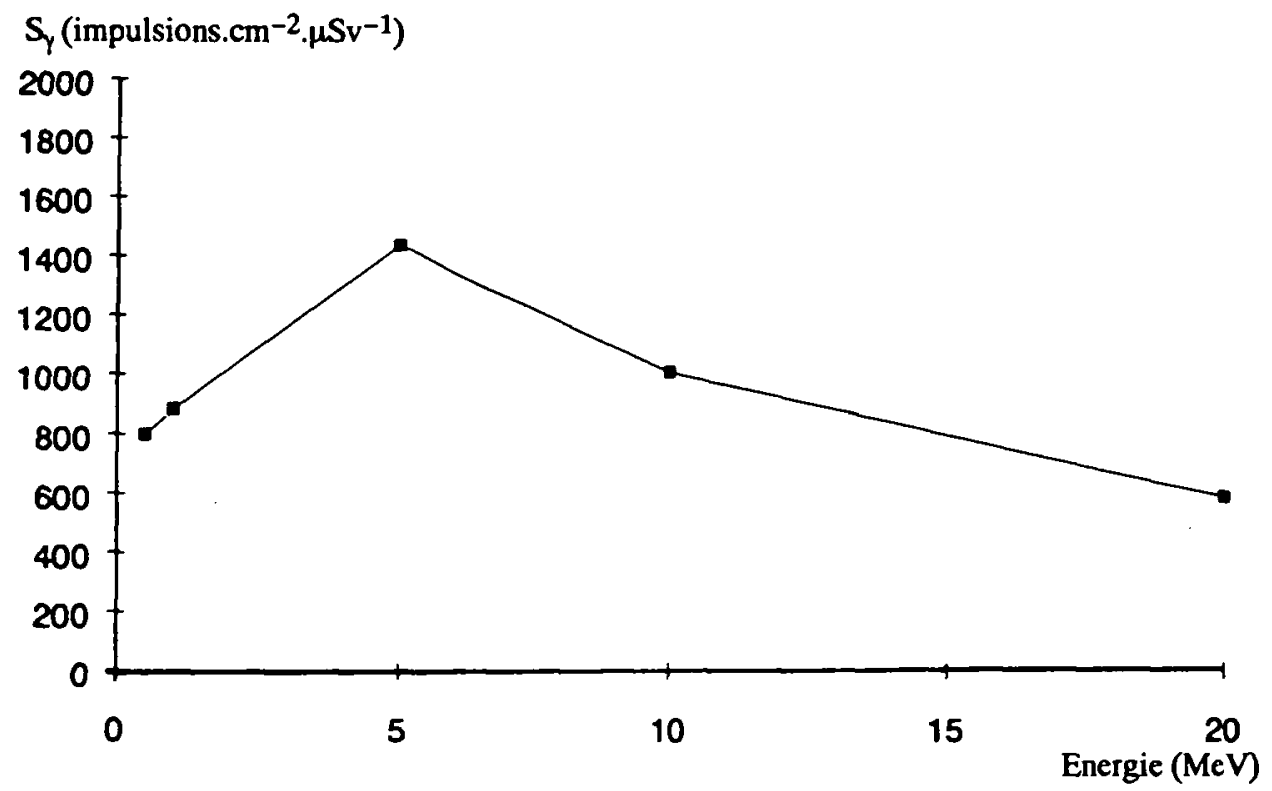

Fig. 3. - Sensibilité théorique en fonction de l'énergie des photons $\gamma$ Profondeur de zone désertée de la diode = 30 um (pas de seull de coupure).

Computed sensitivity vs photon energy $E_{\gamma}$; depth depleted layer detector $=30 \mu \mathrm{m}$ (without energetic threshold).

Nbre d'impulsions/canal

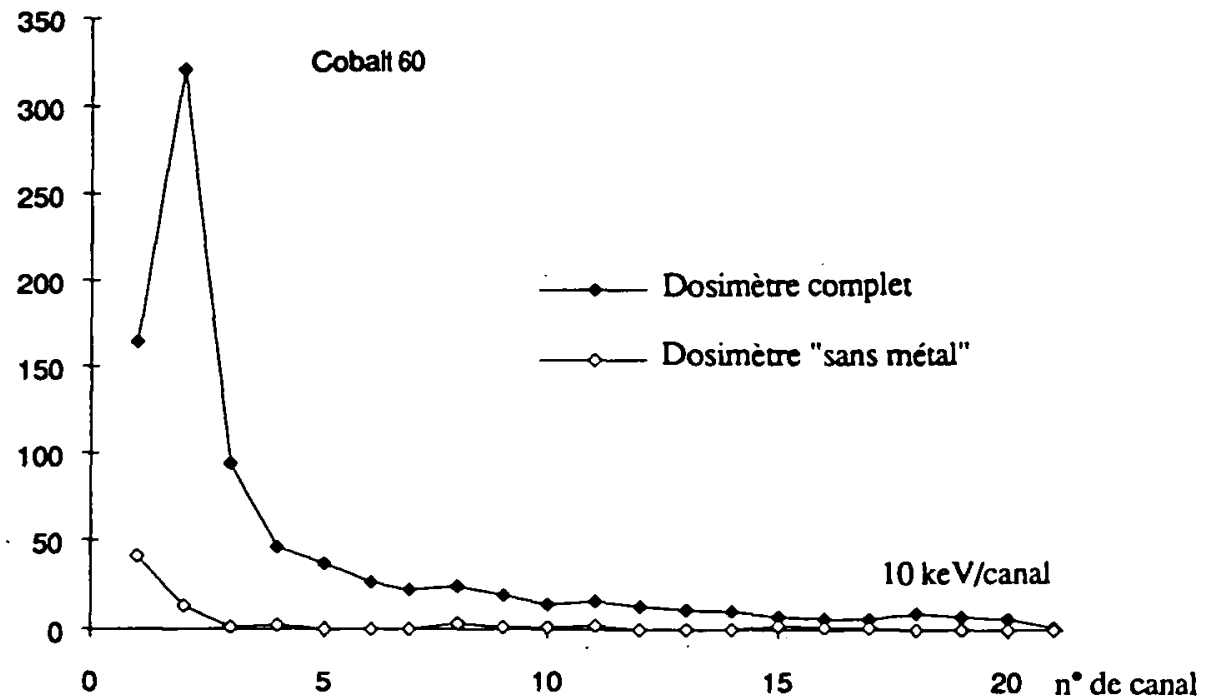

Fig. 4. - Modification théorique d'un spectre dô à une irradlation en ${ }^{60}$ Co par les composants métalliques du dosimètre, $H=0,597 \mu S v$.

Spectrum computed modification due to ${ }^{60} \mathrm{Co}$ irradiation by the dosemeter metallic components, $H=0.597 \mu \mathrm{Sv}$.

\section{Réponse du système dans divers champs de neutrons}

Notre objectif est la réalisation d'un système de mesure délivrant une réponse quasiment indépendante de l'angle et de l'énergie des neutrons, de telle façon que la grandeur dosimétrique souhaitée s'obtienne par un 
simple comptage des impulsions délivrées par la diode. Ce principe permet de simplifier le traitement du signal. II n'est donc pas nécessaire de réaliser une spectrométrie, c'est-à-dire d'avoir une diode avec une profondeur de zone désertée suffisante pour permettre aux particules secondaires les plus énergétiques de déposer toute leur énergie.

Lors de l'étude de faisabilité, nous avions caractérisé le dispositif avec quelques expérimentations (faisceau de neutrons monoénergétiques, source Am-Be, neutrons thermiques). B. Dubarry-Chabanais a étendu cette étude à l'utilisation de champs "réalistes" afin de connaître précisément son comportement et valider son domaine d'application. Les paramètres pris en compte sont l'énergie des neutrons, l'équivalent de dose (de quelques dizaines de $\mu \mathrm{Sv}$ à quelques $\mathrm{mSv}$ ) et le débit d'équivalent de dose (de la cinquantaine de $\mu S v \mathrm{~h}^{-1}$ à la dizaine de $\mathrm{mSv}^{-1}$ ).

\subsection{Comportement du système dans des champs de neutrons monoénergétiques}

Nous avons utilisés des faisceaux de neutrons avec les énergies suivantes : $144 \mathrm{keV}, 570 \mathrm{keV}, 1,2 \mathrm{MeV}$ et 2,5 MeV. Hormis pour l'énergie de $144 \mathrm{keV}$ où le débit d'équivalent de dose est de $1 \mathrm{mSv} \mathrm{h}^{-1}$, dans les autres cas nous avons $10 \mathrm{mSv} \mathrm{h}^{-1}$. Afin que la composante due aux neutrons diffusés reste négligeable, nous avons travaillé en incidence normale. Pour ces faisceaux, le rapport $\mathrm{H}_{n} / \mathrm{H}_{\gamma}$ est de l'ordre de $10^{3}$. Dans ces conditions, la méthode différentielle est statistiquement valide (les impulsions dues aux photons $\gamma$ peuvent être globalement séparées des impulsions dues aux neutrons) [5]. L'épaisseur du convertisseur en $\left(\mathrm{CH}_{2}\right)_{n}$ utilisé $(35 \mu \mathrm{m})$ permet théoriquement d'obtenir une sensibilité $\mathrm{S}_{n}$ d'environ 1 impulsion $\mathrm{cm}^{-2} \mu \mathrm{Sv}^{-1}$ sur une gamme d'énergie de $200 \mathrm{keV}$ à $5 \mathrm{MeV}$ [3].

Les résultats expérimentaux obtenus sont normalisés à un équivalent de dose de $1 \mathrm{mSv}$. A titre d'exemple, l'allure des spectres énergétiques délivrés par les deux voies de mesure du système est représentée (pour $E_{n}=1,2 \mathrm{MeV}$ ) sur la figure 5 , ainsi que la réponse différentielle obtenue par soustraction, canal par canal, des spectres précédents.

\section{a) Détermination du seuil en énergie, réponse en énergie}

Les caractéristiques des détecteurs utilisés n'étant pas rigoureusement identiques, un seuil de coupure électronique distinct a été introduit de manière à obtenir un temps mort identique lors des acquisitions. Malgré ce dernier, certains problèmes d'asymétrie persistent et donc un seuil de coupure énergétique doit être choisi. L'optimisation de ce seuil passe par un compromis entre :

- la sensibilité du détecteur qui doit être la meilleure possible,

- la réponse du dosimètre la plus indépendante possible de l'énergie des neutrons incidents,

- une erreur sur la mesure minimisée au mieux. 


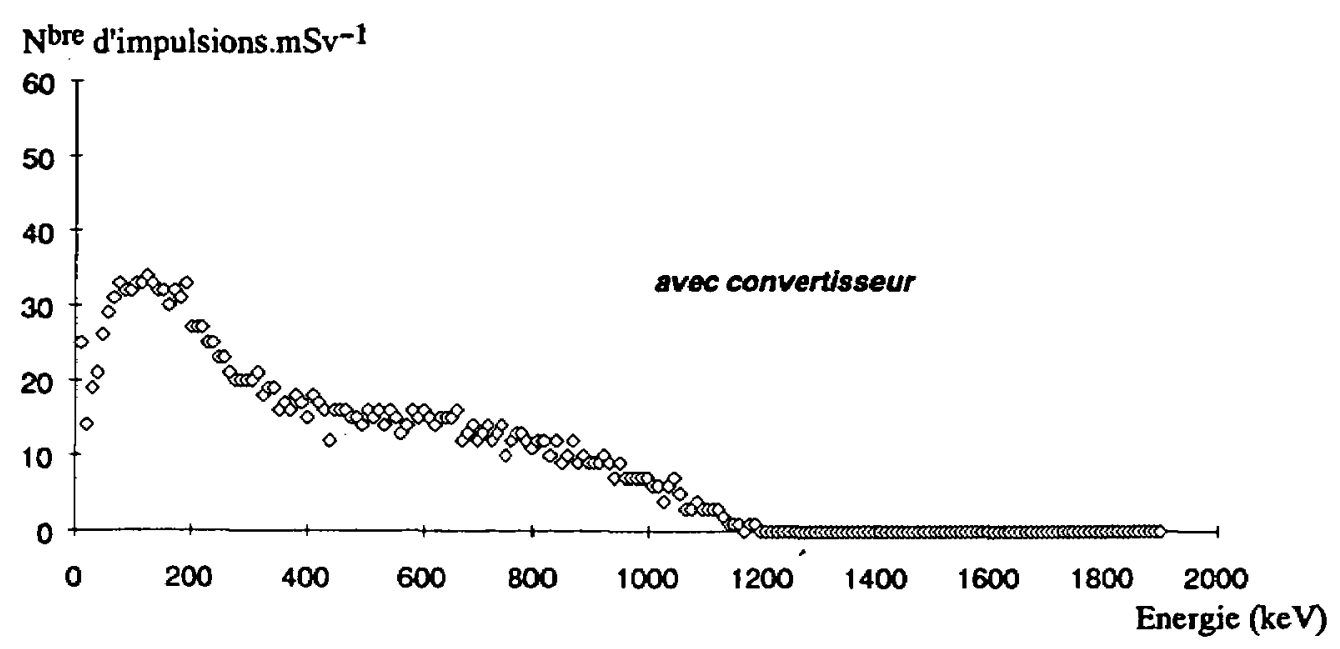

Nbre d'impulsions.mSv-1

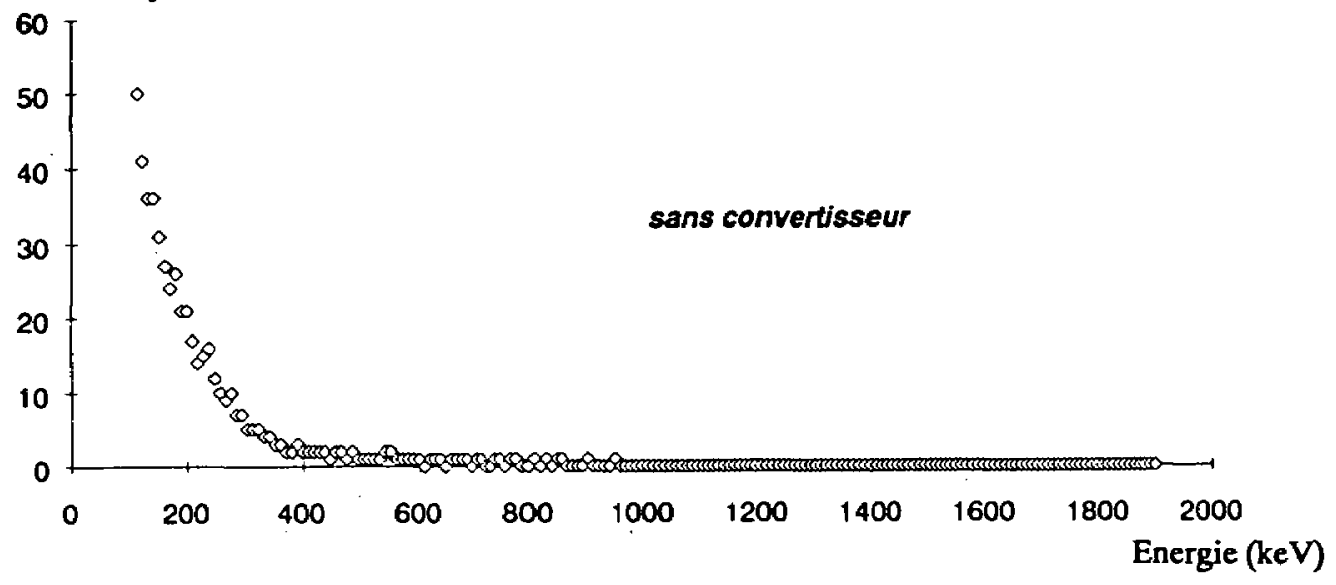

Nbre dimpulsions.mSv ${ }^{-1}$

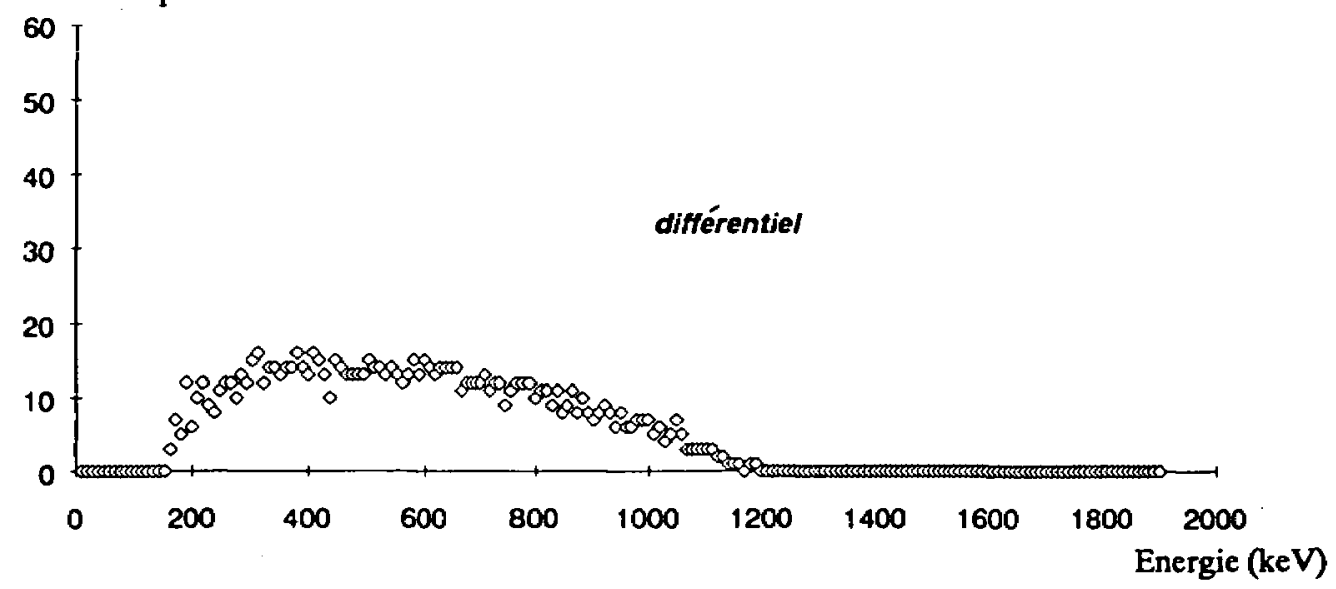

Fig. 5. - Réponse du système à des neutrons de 1,2 $\mathrm{MeV} ; \mathrm{H}=1 \mathrm{mS}$ Sensor response in a $1.2 \mathrm{MeV}$ neutron beam; $H=1 \mathrm{mSv}$.

La prise en compte de ces divers impératifs a permis de fixer une énergie de "coupure" au-delà de laquelle la réponse du détecteur est considérée comme valide (l'erreur que l'on peut introduire en coupant une partie du signal utile est négligeable devant celle que l'on commettrait en comptabilisant des impulsions parasites). 
La figure 6 donne la réponse en fonction de l'énergie pour différents seuils énergétiques. Nous voyons qu'un seuil compris entre le canal 10 (95 keV) et le canal $15(142,5 \mathrm{keV})$ permet de répondre au mieux aux exigences fixées précédemment. Cette indication est confirmée par un calcul de E. Savvidis [12] relatif à l'erreur commise sur le calcul de la différence des intégrales.

D'après ces trois critères (seuil, sensibilité, erreur), il semble raisonnable de choisir un seuil énergétique autour de $125 \mathrm{keV}$ permettant d'obtenir une réponse maximale quelle que soit l'énergie des neutrons, la plus indépendante de leur énergie et dont l'erreur sur le calcul est minimale. A la lumière de ces résultats, l'expérimentation réalisée avec une énergie de neutrons de $144 \mathrm{keV}$ ne sera pas exploitée.

La figure 7 donne la sensibilité $S_{n}$ du système en fonction de l'énergie. Seuls les points à 3,3 et $5,3 \mathrm{MeV}$ ont été obtenus avec des seuils respectifs à 400 et $500 \mathrm{keV}$. Le choix d'un seuil plus élevé n'est pas gênant dans la mesure où la partie du signal due aux protons qui est éliminée est négligeable puisqu'elle se produit aux basses énergies.

La décroissance observée à 1,2 MeV (21\%) et à $570 \mathrm{keV}(47 \%)$ par rapport à la sensibilité moyenne $S_{n}$ sur la gamme 1,2-5,3 $\mathrm{MeV}$ (environ 1 impulsion $\mathrm{cm}^{-2} \mu \mathrm{Sv}^{-1}$ ) s'explique :

- par le fait que le spectre des protons s'étalant moins vers les hautes énergies, le seuil énergétique coupe d'autant plus une partie du signal utile ;

- l'efficacité dosimètrique intrinsèque du convertisseur de $\left(\mathrm{CH}_{2}\right)_{n}$ est moindre pour ces énergies là.

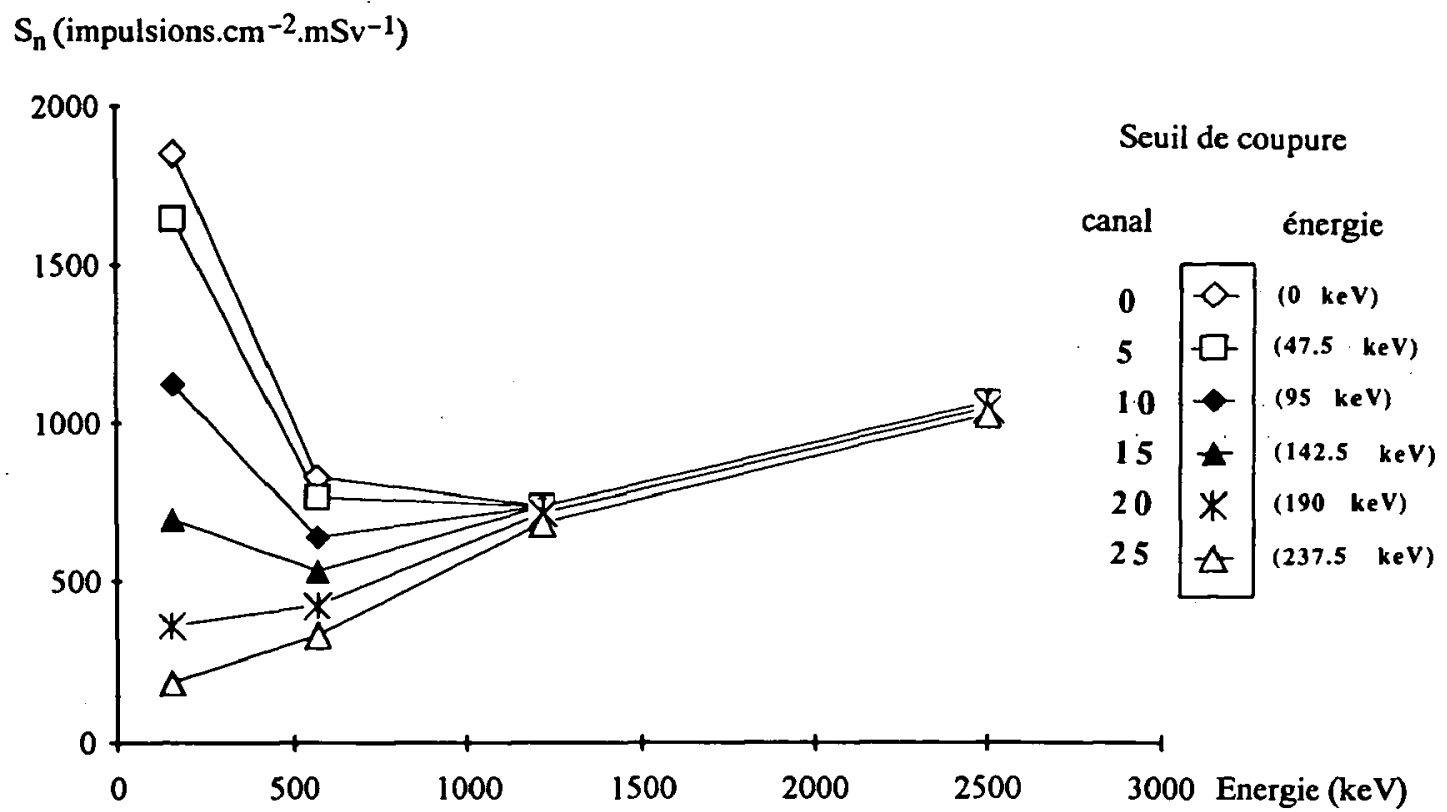

Fig. 6. - Sensibilité $S_{n}$ du système en fonction de l'énergie des neutrons pour divers seuils de coupure.

Neutron sensitivity $S_{n}$ vs neutron energy for various energy thresholds. 
$S_{n}\left(\right.$ impulsions.cm $\left.{ }^{-2} \cdot \mu S v^{-1}\right)$

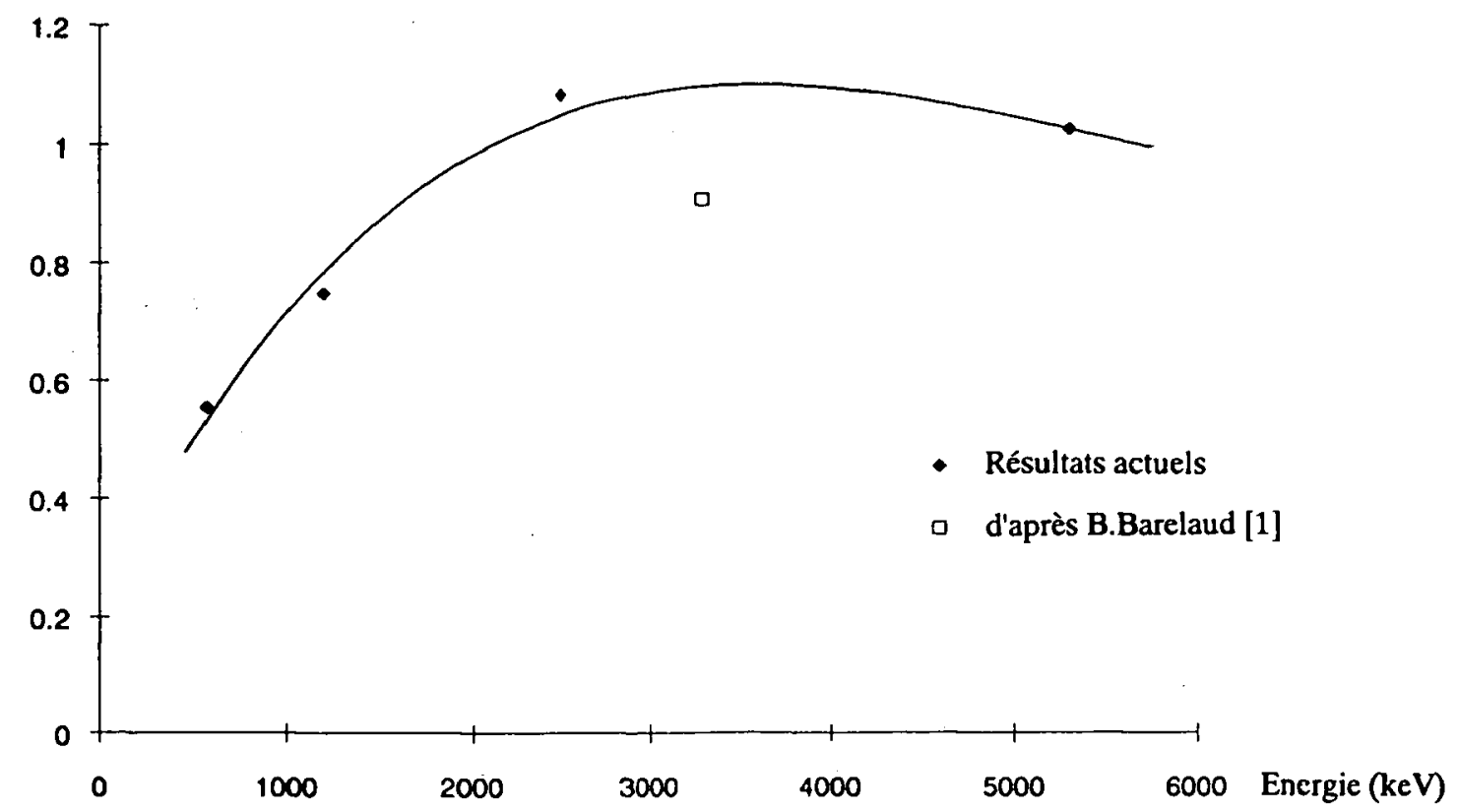

Fig. 7. - Sensibilité $S_{n}$ du système en fonction de l'énergie des neutrons.

Neutron sensitivity $S_{n}$ vs neutron energy.

b) Réponse en fonction de l'équivalent de dose, du débit de dose et de l'angle d'incidence des neutrons

La réponse du système suit une loi linéaire en fonction de l'équivalent en dose, quelle que soit l'énergie (Tab. III).

TABLEAU III

Réponse du système pour plusieurs équivalents de dose et trois énergies de neutrons rapides

Neutron response for various dose equivalent and for three fast neutron energies

\begin{tabular}{|c|c|c|c|}
\hline 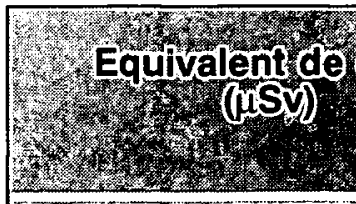 & $70 \mathrm{ke}$ & impul & 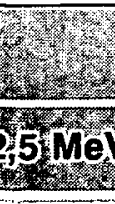 \\
\hline 50 & 23 & 26 & 69 \\
\hline 100 & 32 & 64 & 103 \\
\hline 500 & 217 & 383 & 531 \\
\hline 1000 & 504 & 749 & 1111 \\
\hline 2000 & 976 & 1564 & 2215 \\
\hline
\end{tabular}


Concernant le débit d'équivalent de dose, le tableau IV montre clairement une réponse indépendante dans la gamme proposée pour des neutrons d'énergies $1,2 \mathrm{MeV}$.

TABLEAU IV

Sensibllité $S_{n}$ du système en fonction du débit d'équivalent de dose Neutron sensitlvity $S_{n}$ for various dose equivalent rates

\begin{tabular}{|cccc|}
\hline Débit d'équivalent de dose $\left(\mathrm{mSv} \mathrm{h}^{-1}\right)$ & 1,1 & 4 & 12 \\
\hdashline Sensibilité $\mathrm{S}_{\mathrm{n}}$ (impulsions $\left.\mathrm{cm}^{-2} \mu \mathrm{Sv}^{-1}\right)$ & 0,779 & 0,772 & 0,754 \\
\hline
\end{tabular}

La figure 8 montre la sensibilité relative du détecteur en fonction de l'angle d'incidence des neutrons, pour les énergies testées. II apparaît que la dépendance angulaire est d'autant plus prononcée que l'énergie des neutrons est faible. Ce phénomène a deux origines:

- lorsque l'angle d'incidence des neutrons augmente, les impulsions créées dans le détecteur sont, pour la plus grande partie, issues de particules moins énergétiques et s'enregistrent dans des canaux d'énergie inférieure ;

- d'autre part, les facteurs de conversion fluence-équivalent de dose $H^{\star}(10) / \Phi$ sont des fonctions non seulement de l'énergie mais aussi de la direction d'incidence du faisceau de neutrons; en effet, le facteur de conversion diminue quand l'angle d'incidence augmente [8]; le phénomène est plus important au-delà de $60^{\circ}$.

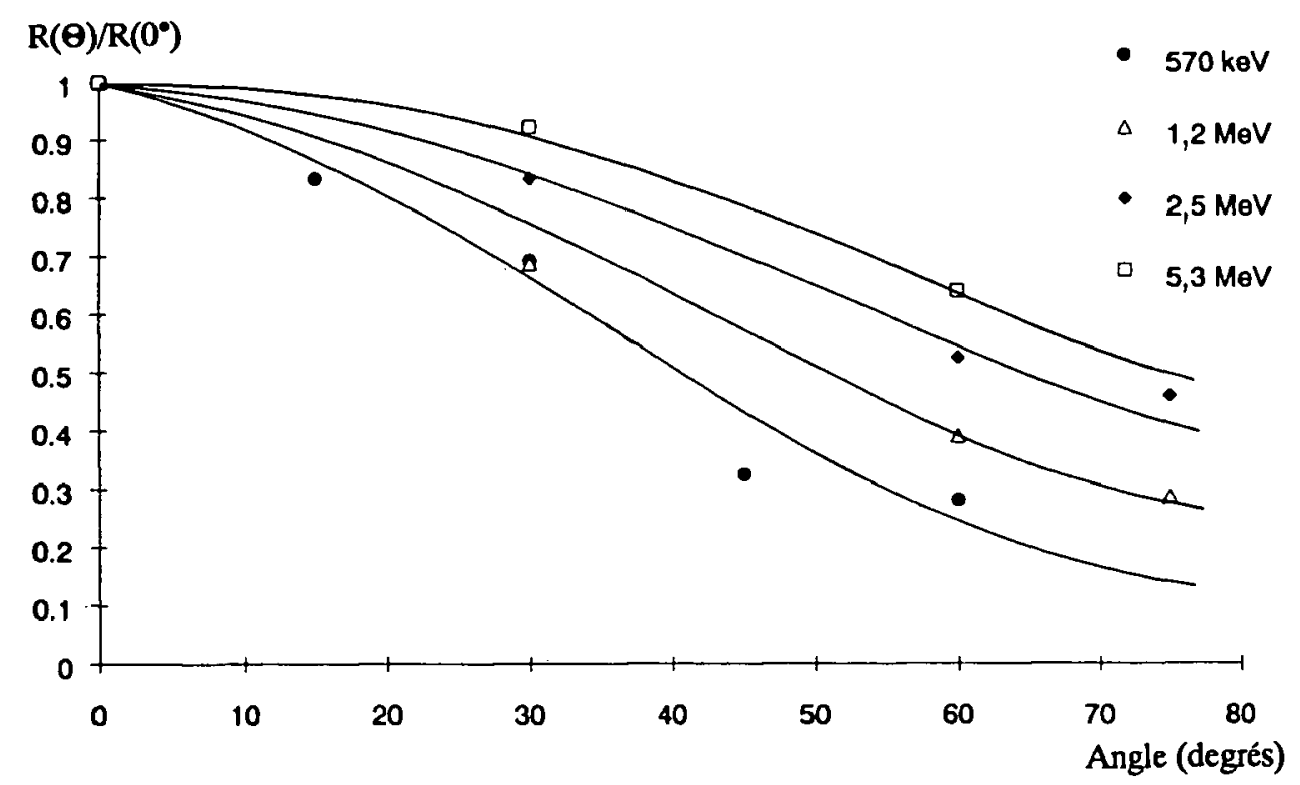

Fig. 8. - Senslbilité relative du système en fonction de l'angle d'incidence $(\Theta)$ des neutrons. Relative sensitivity vs neutron incldent angle $(\Theta)$. 
En première approximation, les calculs réalisés pour estimer l'équivalent de dose dans nos expériences ont été considérés avec des facteurs conversion constants. Les valeurs déduites de ces calculs ont tendance à sous-estimer la réponse de notre détecteur d'autant plus que l'énergie des neutrons est faible. On peut, par conséquent, penser que la dépendance angulaire réelle de la sensibilité du détecteur est moins prononcée.

\subsection{Comportement du système irradié avec des sources de neutrons (AmBe et PuC) et des champs de neutrons réalistes}

Les spectres énergétiques de la source d'AmBe et de PuC s'étendent respectivement de 0 à $11 \mathrm{MeV}$ et de 0 à $8 \mathrm{MeV}$ avec une énergie la plus probable se situant autour de $4 \mathrm{MeV}$ pour les deux sources. Nous avons laissé le système sous irradiation pendant un temps relativement long $(>10 \mathrm{~h}$ ) afin d'obtenir une statistique correcte. Dans ce cas, compte tenu du bruit de fond, le choix d'un seuil de coupure à $350 \mathrm{keV}$ provient d'un bon compromis entre une sensibilité $S_{n}$ la plus importante possible compatible avec une erreur relative suffisamment faible. Dans ces conditions, la sensibilité obtenue pour ces mesures est de 0,750 impulsions $\mathrm{cm}^{-2} \mu \mathrm{Sv}^{-1}$ pour l'irradiation avec la source d'AmBe et de 0,660 impulsions $\mathrm{cm}^{-2} \mu \mathrm{Sv}^{-1}$ pour l'irradiation avec la source de PuC.

Nous avons constaté sur les deux spectres obtenus la présence d'un nombre d'impulsions non négligeables dans le domaine d'énergie 5-6 MeV sur les deux voies (avec et sans convertisseur). Après analyse de différentes hypothèses, nous attribuons cet effet aux interactions directes des neutrons sur le silicium (diffusion élastique) et sur l'aluminium (réactions nucléaires) qui seules peuvent justifier l'existence d'impulsions d'énergie élevée.

Le dosimètre étudié est destiné à être utilisé en radioprotection, c'està-dire auprès d'installations autour desquelles règnent des champs de neutrons polyénergétiques. II était donc nécessaire d'étalonner le dosimètre dans des champs dits "réalistes". Le débit d'équivalent de dose $\mathrm{dH}^{*}(10) / \mathrm{dt}$ étant faible (inférieur à $1 \mathrm{mSv} \mathrm{h}^{-1}$ ), les temps d'exposition ont été suffisamment longs (supérieur à $1 \mathrm{~h}$ ) pour obtenir une bonne précision statistique. En contrepartie, la contribution du bruit de fond est importante et, pour déterminer la sensibilité du système, un seuil énergétique à $550 \mathrm{keV}$ a donc été choisi.

Les quatre spectres de faisceaux de neutrons [4] utilisés sont décrits dans le tableau V.

Pour les deux premiers champs de neutrons [7], nous obtenons les sensibilités suivantes :

$$
\begin{aligned}
& -{ }^{252} \mathrm{Cf}+\mathrm{D}_{2} \mathrm{O}: 0,815 \text { impulsions } \mathrm{cm}^{-2} \mu \mathrm{Sv}^{-1} \\
& -{ }^{252} \mathrm{Cf}+\mathrm{D}_{2} \mathrm{O}+\mathrm{Cd}: 0,560 \text { impulsions } \mathrm{cm}^{-2} \mu \mathrm{Sv}^{-1}
\end{aligned}
$$


TABLEAU $\mathrm{V}$

Spectres de faisceaux de neutrons utilisés pour les mesures expérimentales Neutron beam spectra used for experimentations

\begin{tabular}{|ll|}
\hline $\begin{array}{c}\text { Spectre de neutrons } \\
252 \mathrm{Cf}+\mathrm{D}_{2} \mathrm{O}\end{array}$ & Spectre continu allant des neutrons thermiques aux rapides \\
\hline $252 \mathrm{Cf}+\mathrm{D}_{2} \mathrm{O}+\mathrm{Cd}$ & Spectre continu allant des neutrons épithermiques aux rapides \\
$\mathrm{Canel}+$ & $\begin{array}{l}\text { Spectre de fission dégradé autour de } 300 \mathrm{keV} \\
\text { avec une composante thermique et épithermique } \\
\text { et un pic résiduel à } 14,7 \mathrm{MeV}\end{array}$ \\
Canel+ avec écran $\mathrm{H}_{2} \mathrm{O}$ & $\begin{array}{l}\text { Spectre identique au précédent avec une atténuation } \\
\text { de la composante à } 300 \mathrm{keV} \text { et du pic résiduel à 14,7 } \mathrm{MeV} \\
\text { et une augmentation de la composante thermique } \\
\text { et épithermique }\end{array}$ \\
\hline
\end{tabular}

L'écart entre les deux valeurs peut s'expliquer en partie par : mium,

- la suppression de la composante thermique avec l'écran de cad-

- le décalage vers les basses énergies du pic principal de la distribution énergétique des neutrons (1,3 MeV au lieu de 2,3 MeV avec l'écran d'eau lourde seul) ; le spectre d'impulsions se trouve alors d'autant plus tronqué, en appliquant un seuil à $550 \mathrm{keV}$, qu'il s'enregistre vers les plus basses énergies.

Pour les deux expériences réalisées avec les spectres de neutrons délivrés par "Canel+" [4], nous obtenons les sensibilités suivantes :

- "Canel+" : 0,300 impulsions $\mathrm{cm}^{-2} \mu \mathrm{Sv}^{-1}$,

- "Canel+" $\mathrm{H}_{2} \mathrm{O}: 0,170$ impulsions $\mathrm{cm}^{-2} \mu \mathrm{Sv}^{-1}$.

Cette faible sensibilité s'explique par le fait que d'une part notre dispositif est "aveugle" pour la composante neutronique à $300 \mathrm{keV}$ qui représente plus de $50 \%$ du flux de neutrons. Les réponses spectrales obtenues sont donc composées essentiellement des protons de recul induits par le pic résiduel neutronique à $14,7 \mathrm{MeV}$ et les particules $\alpha$ ou ions ${ }^{7} \mathrm{Li}$ nés des réactions nucléaires des neutrons thermiques sur le bore 10. D'autre part, le seuil énergétique fixé à $550 \mathrm{keV}$ pour s'affranchir du bruit de fond coupe une grande partie de cette réponse. Enfin, l'écart de sensibilité entre les deux étalonnages provient de l'atténuation du pic résiduel par l'écran d'eau.

\subsection{Conclusion de l'étude expérimentale}

Pour les faisceaux monoénergétiques, nous avons une sensibilité moyenne :

$$
\begin{gathered}
S_{n}=0,851 \pm 0,247 \text { impulsions } \mathrm{cm}^{-2} \mu S v^{-1} \\
\text { avec un seuil fixé à } 125 \mathrm{keV}^{-1}
\end{gathered}
$$


Pour les faisceaux polyénergétiques (sources et champs réalistes excepté "Canel+"), nous obtenons :

$$
\begin{gathered}
S_{n}=0,700 \pm 0,110 \text { impulsions } \mathrm{cm}^{-2} \mu \mathrm{Sv}^{-1} \\
\text { avec un seuil variant de } 350 \text { à } 550 \mathrm{keV}
\end{gathered}
$$

$$
\begin{aligned}
& \text { Pour les champs réalistes "Canel+" : } \\
& \qquad \begin{array}{c}
S_{n}=0,250 \pm 0,113 \text { impulsions } \mathrm{cm}^{-2} \mu \mathrm{Sv}^{-1} \\
\text { avec un seuil fixé à } 550 \mathrm{keV}
\end{array}
\end{aligned}
$$

Le seuil énergétique qui joue de façon importante sur la sensibilité demeure un handicap.

Cette étude expérimentale nous a permis de réaliser un étalonnage relativement complet du dosimètre et d'extraire les zones où ce dernier reste partiellement insensible. La modélisation doit permettre d'optimiser tous les paramètres mis en jeu.

\subsection{Etudes théoriques du capteur et simulation}

A l'heure actuelle, il n'existe pas de programme de calcul qui traite l'ensemble des interactions dans un matériau, non seulement des neutrons incidents, mais aussi des particules chargées résultant de l'interaction des neutrons. C'est pourquoi nous avons développé notre propre programme en nous basant sur les premiers calculs réalisés au LEPOFI. La modélisation du passage des neutrons rapides à travers un milieu organique réalisée par L. Makovicka nous a plus particulièrement servi de base d'étude [8]. En effet, les réponses énergétique et angulaire d'un convertisseur en polyéthylène ont été étudiées dans le cas des détecteurs CR 39.

Nous avons appliqué le principe de cette méthode au cas particulier de notre capteur électronique, c'est-à-dire à un convertisseur $\left(\mathrm{CH}_{2}\right)_{n}$ de $35 \mu \mathrm{m}$, implanté en ${ }^{10} \mathrm{~B}$, associé au détecteur silicium. Ainsi, nous avons simulé les pertes d'énergie des particules chargées à travers la zone désertée du détecteur et remonter, par comptage et classement en énergie, aux spectres observés expérimentalement. Un des principaux avantages de la simulation par rapport aux mesures est la discrimination parfaite des impulsions, connaissant les particules qui leur ont donné naissance, et donc l'évaluation de chaque composante du spectre de particules chargées.

Dans ces premiers calculs, seuls les produits de la diffusion élastique ont été pris en compte. Afin d'élaborer notre nouveau programme de calcul, nous avons retenu parmi les différentes interactions possibles des neutrons sur les matériaux mis en jeu (polyéthylène, bore 10, Si) celles qui étaient prépondérantes. En réalité, seules les diffusions élastiques et les réactions nucléaires $(n, p)$ et $(n, \alpha)$ sont prises en compte dans notre modèle ; les réactions ne fournissant pas de particules chargées $[(n, 2 n)$ et $(n, \gamma)]$ sont aussi écartées de la simulation. 
La modélisation du capteur, utilisée pour l'étude en photons $\gamma$, est conservée pour l'étude en neutrons. La figure 9 montre schématiquement une diode CANBERRA modélisée avec son boîtier. Pour une première étude du capteur, nous avons négligé d'une part l'absorption des neutrons due à la plaque métallique placée à l'avant et d'autre part tous les matériaux situés à l'arrière de la zone silicium et sur les côtés. Seuls sont considérés les éléments intervenant directement au niveau de la détection, à savoir le convertisseur en $\left(\mathrm{CH}_{2}\right)_{n}$ implanté sur sa face arrière avec des atomes de bore 10, et le substrat de silicium constitué des zones avant (zone morte), intermédiaire (zone désertée) et arrière (zone de type N).

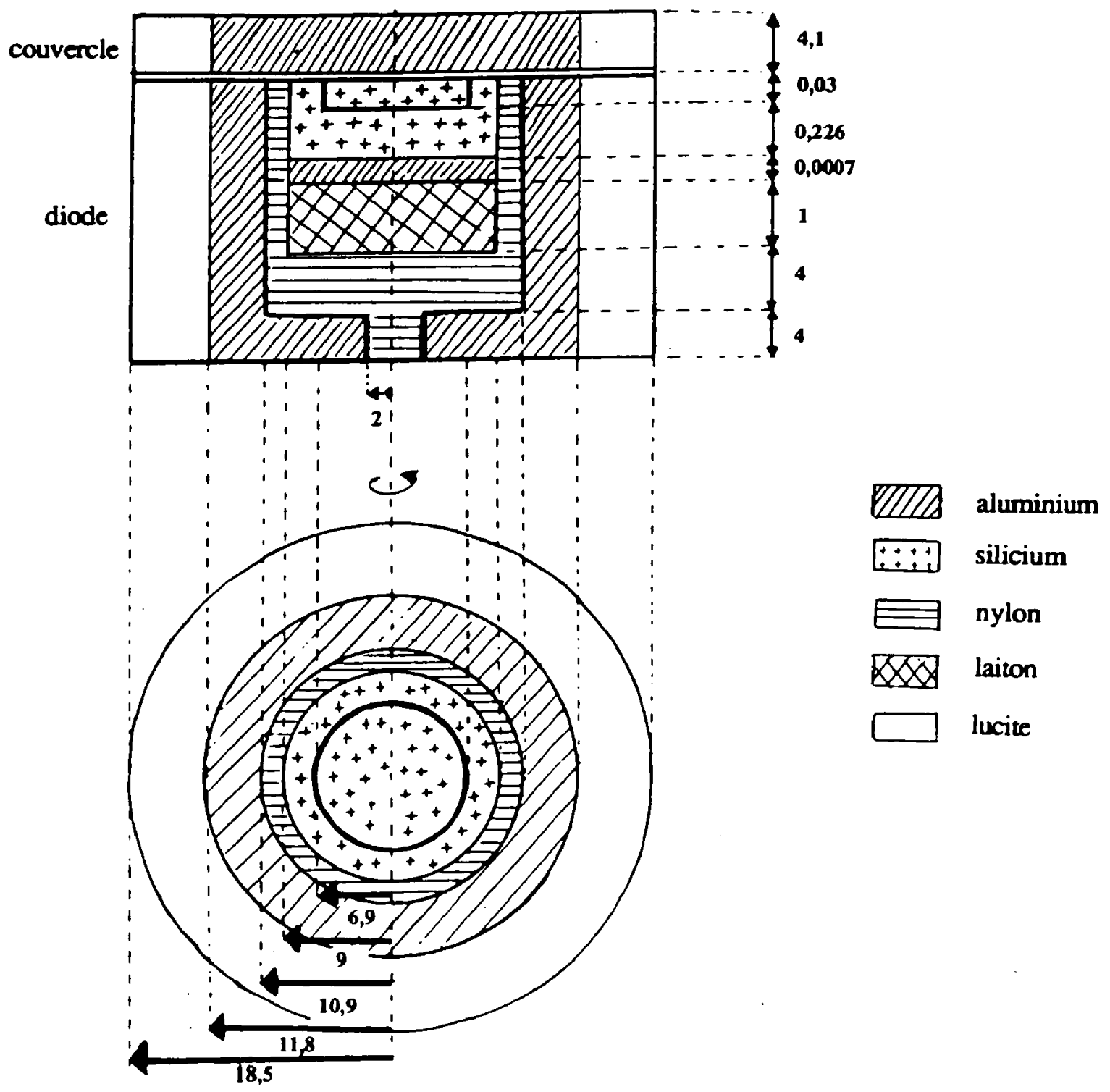

Fig. 9. - Schéma d'une diode CANBERRA et de son boîtier (cotes en $\mathrm{mm}$ ) Schematic view of a CANBERRA diode with its case (size in $\mathrm{mm}$ ) 


\section{a) Résultats de la simulation}

Les premiers résultats de la simulation nous ont permis de comparer les sensibilités $S_{n}$ théoriques et expérimentales ainsi que les distributions énergétiques. Le tableau $\mathrm{VI}$ donne un aperçu de ces comparaisons. Malgré les hypothèses simplificatrices apportées au niveau du programme de calcul, nous voyons que l'accord entre les diverses expériences dans des champs de neutrons et les premiers résultats obtenus par simulation est relativement bon.

TABLEAU VI

Comparaison des sensibilités théorique et expérimentale $S_{n}$ du capteur dans divers champs de neutrons

Experimental and computed sensor sensitivities $\mathbf{S}_{\mathbf{n}}$ in various neutron fields

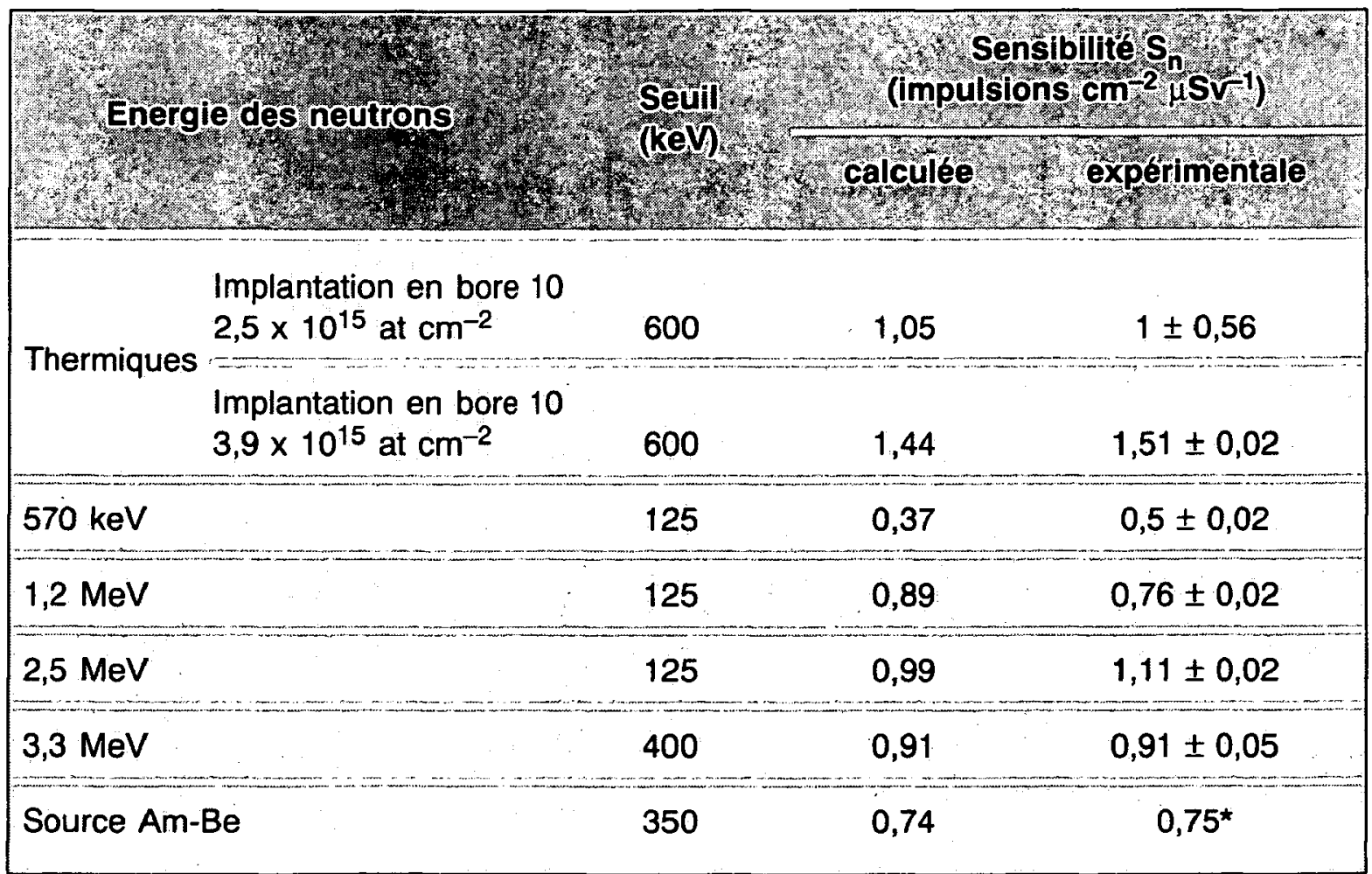

* Résultat correspondant à une seule mesure.

Les figures $10 \mathrm{a}$ et $10 \mathrm{~b}$ montrent aussi une bonne concordance pour les distributions énergétiques.

Par la suite, nous avons effectué d'autres simulations pour d'une part fixer les limites de validité du programme de calcul (tests dans différentes configurations du faisceau de neutrons incidents) et d'autre part envisager une optimisation des caractéristiques du capteur en faisant varier divers paramètres (épaisseur du convertisseur...). Pour trois énergies - $500 \mathrm{keV}, 1 \mathrm{MeV}$ et 2,5 MeV - nous avons vérifié que la réponse obtenue est bien linéaire en fonction de l'équivalent de dose dans la gamme de $30 \mu \mathrm{Sv}$ à $3 \mathrm{mSv}$ et que la statistique utilisée pour ces calculs est suffisante. Les tests de répétabilité effectués avec le programme de simulation donnent des écarts de $0,1 \%$. 

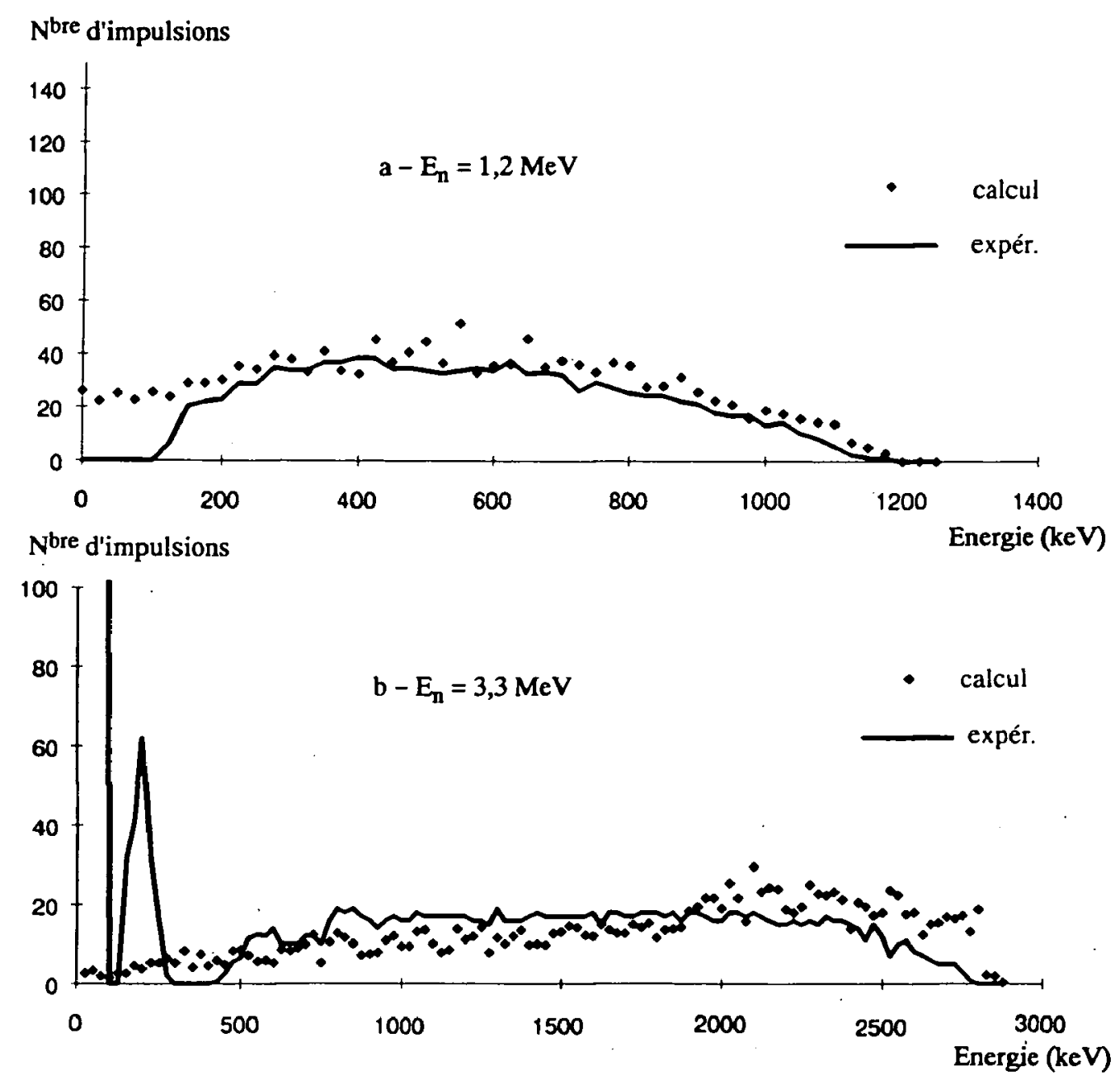

Fig. 10. - Spectres des impulsions théoriques et expérimentales dues aux protons : a) $E_{n}=1,2 \mathrm{MeV}$; b) $E_{n}=3,3 \mathrm{MeV}$.

Computed and experimental spectra due to recoil protons : a) $E_{n}=1.2 \mathrm{MeV}$; b) $E_{n}=3.3 \mathrm{MeV}$.

\section{b) Variation des paramètres du modèle}

Nous avons étudié la variation de sensibilité du capteur en fonction de l'épaisseur du convertisseur de $\left(\mathrm{CH}_{2}\right)_{n}$ et de la profondeur de la zone désertée du détecteur. La figure 11 montre qu'avec une épaisseur de convertisseur de $25 \mu \mathrm{m}$, on réduit la dispersion des valeurs de sensibilité en fonction de l'énergie. Cette possibilité d'accroître l'indépendance énergétique de la sensibilité $S_{n}$ se fait au détriment de cette dernière. Pour des profondeurs de zone désertée variant, les résultats des calculs ont montré une indépendance de la sensibilité $S_{\mathrm{n}}$ du capteur de $10 \mu \mathrm{m}$ à $80 \mu \mathrm{m}$ et ce quelle que soit l'énergie des neutrons de $150 \mathrm{keV}$ à $8 \mathrm{MeV}$. 


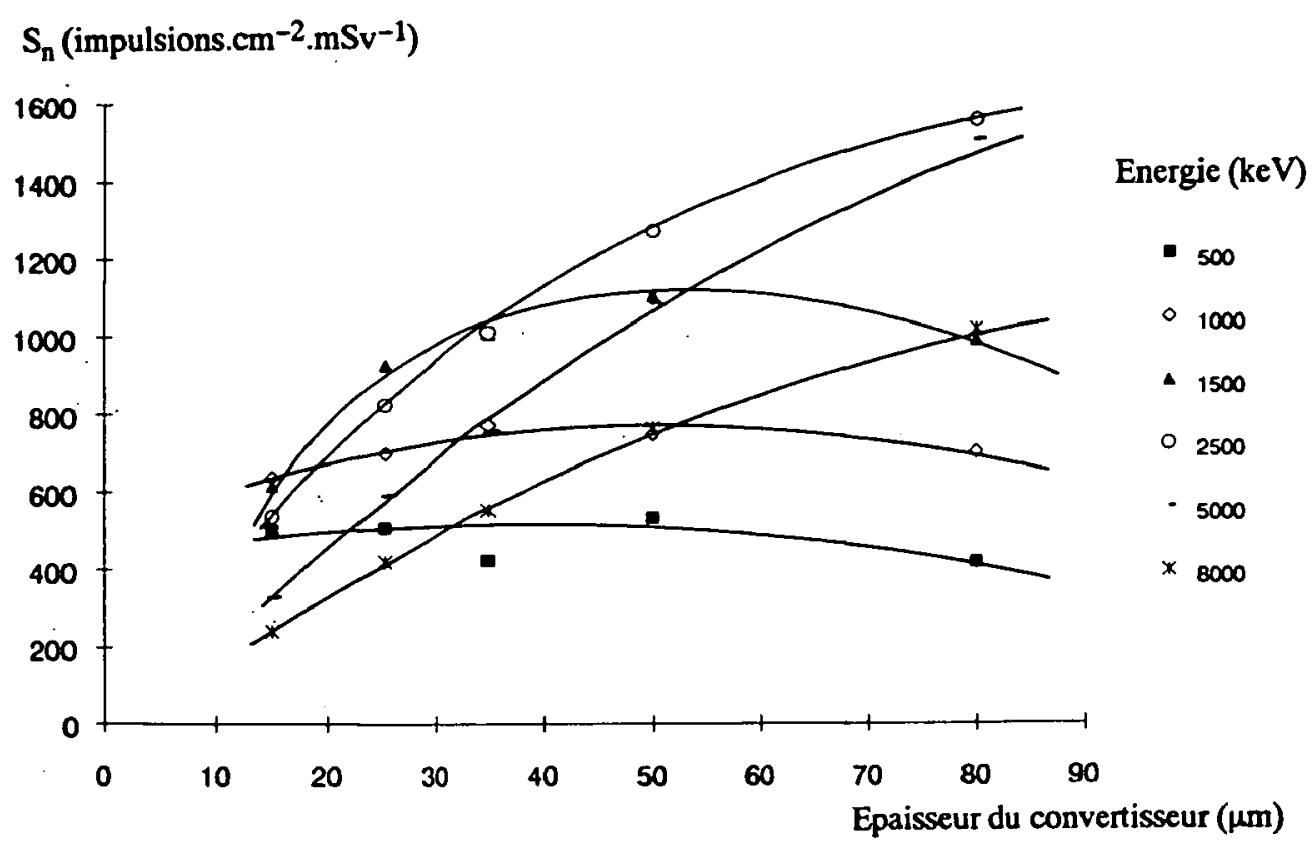

Fig. 11. - Sensibilité $S_{n}$ du capteur calculée en fonction de l'épaisseur de convertisseur, pour diverses énergles de neutrons incidents (Incidence normale).

Computed sensor sensitivity $S_{n}$ vs converter thickness for different neutrons energles (normal Incidence).

\section{Comparaison des calculs avec les réponses du capteur de l'étude de faisabilité et du capteur actuel}

Le tableau VII nous permet de suivre l'évolution des performances du capteur, principalement pour :

- la sensibilité $S_{\gamma}$ et l'étalement du spectre,

- les équivalents de dose et de débit minimaux détectés, tiques.

- le seuil de coupure énergétique pour les neutrons monoénergé-

\section{Conclusions}

Au terme de ce travail, nous disposons d'un système électronique de dosimétrie neutronique mieux connu et plus performant que le dosimètre développé à l'origine au laboratoire. Les premières améliorations ont porté sur des considérations purement pratiques; elles n'étaient pas sous-tendues par des calculs et ont conduit d'abord à l'acquisition de nouvelles diodes puis à la révision de la technique d'étalonnage des voies de mesure. Les calculs et expériences menés en parallèle concernent toujours un dosimètre à l'état de "macromontage", mais qui, avec les évolutions subies par rapport aux premières propositions, peut être qualifié de "deuxième génération". 
TABLEAU VII

Principales caractéristiques du capteur utilisé lors de l'étude de falsabilité et du capteur actuel

Main sensor characteristics used during the feasability study and presently

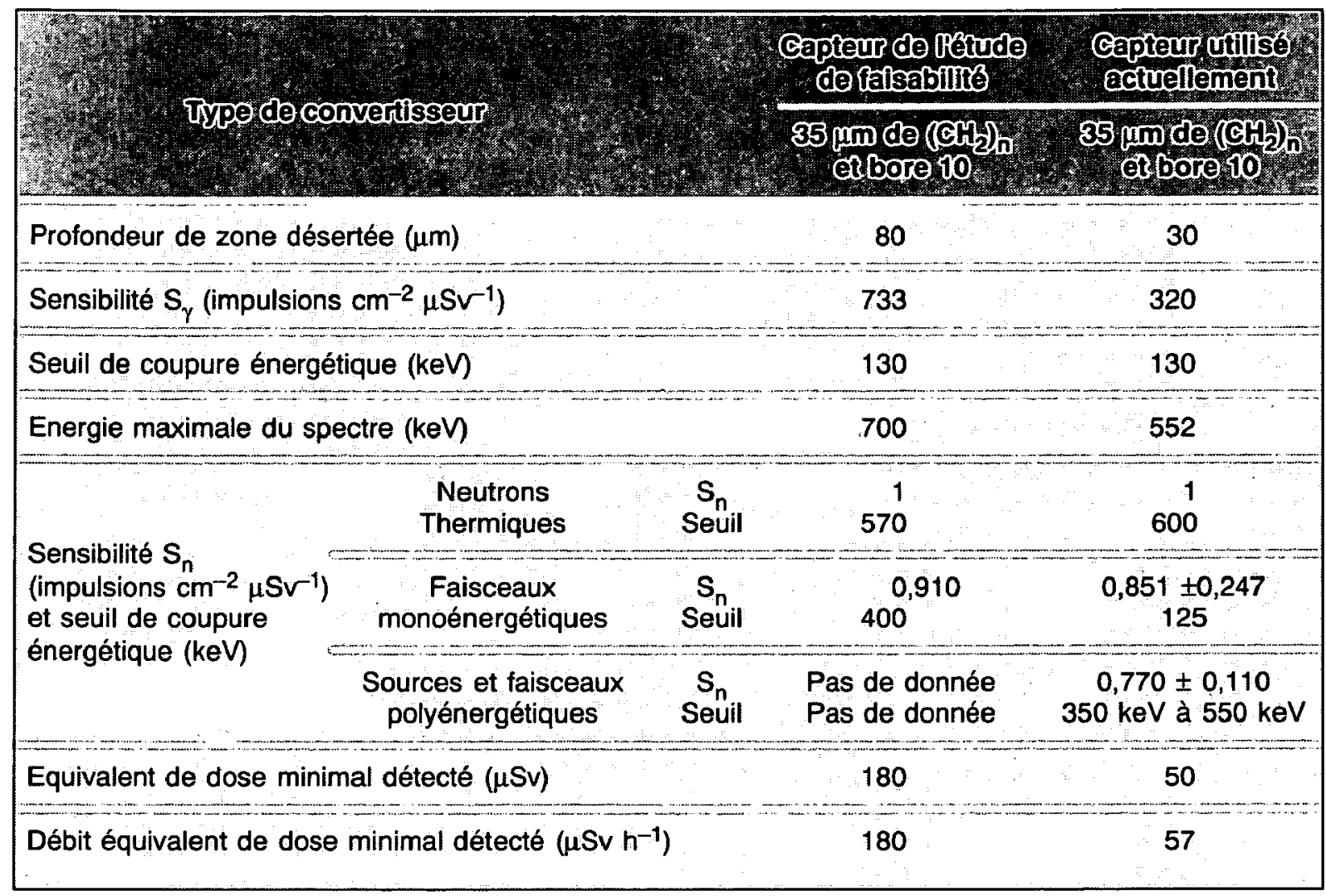

Les résultats acquis aussi bien par simulation que par expérimentation ont permis de situer les performances actuelles du système.

En photons $\gamma$, nous pouvons les résumer de la façon suivante :

- la sensibilité $S_{\gamma}\left({ }^{60} \mathrm{Co}\right.$ et $\left.{ }^{137} \mathrm{Cs}\right)$ est de l'ordre de 320 impulsions $\mathrm{cm}^{-2} \mu \mathrm{Sv}^{-1}$ avec un seuil énergétique de coupure à $130 \mathrm{keV}$ et une énergie maximale du spectre enregistré de $552 \mathrm{keV}$; cette sensibilité reste malgré tout 320 fois supérieure à celle des neutrons ;

- nous n'avons pas observé d'indépendance énergétique et angulaire de la sensibilité mais un maximum de celle-ci autour de $E_{\gamma}=E \mathrm{MeV}$ et une perte de réponse pour les incidences $\gamma$ obliques $\left(45^{\circ}\right)$;

- parmi les éléments qui enveloppent la zone de détection, le convertisseur en polyéthylène joue un rôle faible, par contre la plaque d'entrée et le boîtier en aluminium se comportent en "générateurs" d'électrons plutôt qu'en atténuateurs de photons ;

- dans l'état actuel, le système possède un seuil de discrimination $(n-\gamma)$ voisin de $300 \mathrm{keV}$ : au-delà de cette valeur, le nombre d'impulsions dues aux $\gamma$ est du même ordre de grandeur que le nombre d'impulsions dues aux neutrons ; la méthode différentielle devient alors applicable. 


\section{En neutrons :}

- la sensibilité $S_{n}$ pour les faisceaux monoénergétiques de $570 \mathrm{keV}$ à $14 \mathrm{MeV}$ est de 0,85 $\pm 0,25$ impulsions $\mathrm{cm}^{-2} \mu \mathrm{Sv}^{-1}$ avec un seuil de coupure énergétique de $125 \mathrm{keV}$; pour les sources et les champs polyénergétiques nous avons $S_{n}=0,77 \pm 0,11$ impulsions $\mathrm{cm}^{-2} \mu \mathrm{Sv}^{-1}$ avec un seuil compris entre 350 et $550 \mathrm{keV}$;

- l'équivalent de dose minimal a été enregistré avec des neutrons de $570 \mathrm{keV}, 1,2 \mathrm{MeV}$ et $2,5 \mathrm{MeV}$, il est égal à $50 \mu \mathrm{SV}$; cette valeur, pour faible qu'elle soit, répond encore mal à l'ambition des recommandations de la CIPR 60, d'autant plus que le débit d'équivalent de dose minimal (actuellement pour le système) a été mesuré à $57 \mu \mathrm{Sv} \mathrm{h}^{-1}$, avec des neutrons thermiques.

En comparaison, on peut dire très grossièrement que les nouvelles recommandations amènent à mesurer au pire environ $250 \mu \mathrm{Sv}$ par jour (équivalent de dose maximal autorisé de $50 \mathrm{mSv}$ par an pour les personnels). En admettant que la sensibilité recommandée du dispositif soit inférieure ou égale au dixième de la valeur la plus faible à mesurer, on s'aperçoit que, comme la plupart des dosimètres à neutrons actuels, ce système exige des améliorations.

Enfin, l'analyse de tous ces résultats permet d'envisager une évolution générale de la structure du dispositif. Pour le dosimètre de "troisième génération", nous proposons une modification de la structure extérieure au détecteur; on peut citer :

- une diminution de la zone désertée : ce paramètre qui n'a pas d'influence sur la sensibilité $S_{n}$ aux neutrons doit être fixé par un compromis optimisant la profondeur afin de réduire la sensibilité $S_{\gamma}$ aux photons $\gamma$ au minimum et à garder une capacité de jonction faible (problème de bruit électronique) ;

- l'adjonction d'une couche de carbone sur le détecteur sans convertisseur pour éliminer par la méthode différentielle les électrons et les atomes de recul provenant du polyéthylène ;

- la diminution de l'épaisseur, voire la suppression de la plaque d'entrée en aluminium ;

- le remplacement du boîtier en aluminium par un composé organique ad'hoc ; ces modifications doivent permettre une réduction de sensibilité aux photons $\gamma$ d'un facteur 15 et par voie de conséquence un seuil de discrimination $(n-\gamma)$ inférieur à $200 \mathrm{keV}$.

Dans un futur proche, il nous semble que le dosimètre électronique individuel pour les neutrons répondant aux recommandations de la CIPR 60 devrait être un système possédant un capteur multiplage de manière à augmenter la sensibilité aux neutrons $S_{n}$ et à réaliser la discrimination $(n-\gamma)$. Le LEPOFI espère bien pouvoir proposer ce dosimètre de "quatrième génération".

Remerciements : la majeure partie du travail expérimental a pu être réalisé grâce aux irradiations obtenues auprès des centres d'études nucléaires de Bruyères-le-Châtel, 
Cadarache et Fontenay-aux-Roses que nous remercions. L'ensemble de cette étude a été soutenu par la Commission des communautés européennes à travers le contrat B/700200C.

\section{RÉFÉRENCES}

[1] BARELAUD B. - Conception et réalisation d'un capteur pour les neutrons thermiques et rapides. Thèse $n^{\circ} 7-89$, Université de Limoges, 1989.

[2] BARELAUD B., DECOSSAS J.L., MAKOVICKA L., VAREILLE J.C. - Capteur électronique pour la dosimétrie des neutrons. Radioprotection, 1991, 26, 307-328.

[3] BARELAUD B. - Principles of an electronic neutron dosemeter using a PIPS detector. réf. 3 et 4 . In : 7. Symposium on neutron dosimetry, Berlin, 14-18 October 1991. Radiat. Prot. Dosim., 1992, 44 (1/4), 363-366.

[4] CHARTIER J.L., POSNY F., BUXEROLLE M. - Experimental assembly for the simulation of realistic neutron spectra. In : 7. Symposium on neutron dosimetry, Berlin, 14-18 October 1991. Radiat. Prot. Dosim., 1992, 44 (1/4), 125-130.

[5] DUBARRY-CHABANAIS B. - Contribution à l'étude d'un capteur électronique pour la dosimétrie personnelle des neutrons. Thèse $n^{\circ} 1254$, Université Paul Sabatier, Toulouse, 1992.

[6] EISEN Y., ENGLER G., OVADIA E., SHAMAI Y., BAUM Z., LEVY Y. - A small size neutron and gamma dosimeter with a single silicon surface barrier detector. DOE workshop on personnal neutron dosimetry, Acapulco, Mexico. PNL-SA-12352, 1983, 157-175.

[7] KURDJIAN J. - Communication personnelle. CEA/IPSN, Centre d'études de Cadarache.

[8] MAKOVICKA L. - Contribution à la dosimétrie neutron-gamma. Etude d'un ensemble radiateur - détecteur type CR39. Thèse $n^{\circ} 17-87$, Université de Limoges, 1987.

[9] MATSUMOTO T. - PIN diode for real time dosimetry in a mixed field of neutrons and gamma rays. Radiat. Prot. Dosim., 1991, 35 (3), 193-197.

[10] NELSON W.R., HIRAYAMA H., ROGERS D.W.O. - The EGS4 code system. Stanford linear accelerator center, Report SLAC-265, 1985.

[11] PAUL D. - Perturbation gamma dans un dosimètre neutronique à diodes. Thèse $n^{\circ}$ 14-92, Université de Limoges, 1992.

[12] SAVIDIS E. - Communication personnelle. Université Aristotle, Thessalonique (Grèce), 1992. 